\title{
PARAFERMION STATISTICS AND THE APPLICATION TO NON-ABELIAN QUANTUM HALL STATES
}

\author{
EDDY ARDONNE
}

\begin{abstract}
The (exclusion) statistics of parafermions is used to study degeneracies of quasiholes over the paired (or in general clustered) quantum Hall states. Focus is on the $\mathbb{Z}_{k}$ and $\mathfrak{s u}(3)_{k} / \mathfrak{u}(1)^{2}$ parafermions, which are used in the description of spin-polarized and spin-singled clustered quantum Hall states.
\end{abstract}

\section{INTRODUCTION}

In the last decade, low-dimensional systems in which the fundamental (quasi) particles do not satisfy bose or fermi statistics have received a lot of attention. Among the most famous examples are the fractional quantum Hall $(\mathrm{fqH})$ systems. The quantum Hall states at simple filling fractions $\nu=\frac{1}{M}$, where $M$ is an odd integer, are understood in terms of the famous Laughlin states [1]. Quasiparticles (or quasiholes) over these states carry fractional charge and satisfy fractional statistics.

In the last few years, generalizations of these states have been under investigation. Among these generalizations are so-called paired (or more general, clustered) quantum Hall states. The notion of clustering will be explained in the next section. In the definition of these states, parafermions play a predominant role. Ultimately, it is the presence of these parafermions which cause the quasiholes to have peculiar statistics properties, namely, they obey 'non-abelian' statistics (see, for an early reference, [2]).

The simplest of the paired quantum Hall states is the pfaffian state proposed by G. Moore and N. Read [2]; it is believed that this states describes the quantum Hall effect at filling fraction $\nu=\frac{5}{2}$. This quantum Hall effect is special in the sense that it is the only quantum Hall effect observed at a filling fraction with an even denominator (in single layer samples) [3, 46. For a recent review on this subject, see [5. Clustered analogues of the paired pfaffian state were proposed by N. Read and E. Rezayi (RR) [6]. The states mentioned above are all spin-polarized; spin-singlet analogues were proposed in [7]. Recently, the bosonic versions of the RR states were shown to be relevant in the context of rotating Bose-Einstein condensates 8].

The statistics properties of the parafermion fields will be investigated in this paper, with the intention of obtaining closed form expressions for the ground state degeneracy of clustered quantum Hall states in the presence of quasihole excitations, as described in [9, 10]. This state counting problem is interesting for the following reasons. The clustered quantum Hall states can be seen as ground states of a hamiltonian with an (ultra local) interaction between the electrons. Finding the ground state degeneracy of this hamiltonian can be done in a conformal field theory (CFT) approach, relying heavily on the statistics properties of the parafermionic fields. Another approach is by numerically diagonalizing the interaction hamiltonian for a small number of electrons. This method can serve as a check on the analytical results of the first approach. Thus, the quasihole

Key words and phrases. Parafermions, quantum Hall effect, state counting.

ITFA-01-29. 
degeneracies of a system of interacting electrons can be understood in terms of parafermionic statistics!

In the context of the the spin-polarized states of Read and Rezayi, the $\mathbb{Z}_{k}\left(\right.$ or $\left.\mathfrak{s u}(2)_{k} / \mathfrak{u}(1)\right)$ parafermions are the relevant parafermions. For the 'non-abelian spin-singlet' (NASS) states of [7], the relevant parafermions are the parafermions related to $\mathfrak{s u}(3)_{k} / \mathfrak{u}(1)^{2}$ (see [1] for a discussion on general parafermion CFTs).

The plan of the paper is as follows. We first recall in which way (clustered) quantum Hall states can be constructed in CFTs (section 2). In section 3 we will shortly indicate the setup of numerical diagonalization studies, because we need to adapt the calculations to the setup in which these studies are done. The general structure of the counting formulas will be indicated in section 4. It will become clear that the degeneracy consists of an intrinsic and an orbital part, which need to be combined in the right way. The intrinsic degeneracy factors need to be split to make this possible. The remainder of the paper is devoted to this task, as no explicit expressions for these 'split degeneracies' were known for general level $k>2$ for the states under consideration in this paper. We will explain the procedure to obtain these expressions using the $\mathfrak{s u}(3)_{2} / \mathfrak{u}(1)^{2}$ parafermions of the NASS state at level $k=2$ as an example (see [10]). The first step is to find a basis for the (chiral) spectrum of the parafermion CFT. Using this basis, recursion relations for truncated characters will be derived (section 5). These recursion relations can be solved using the results of section 6, providing expressions for the truncated characters. From the explicit truncated characters, the 'split degeneracies' can be extracted. Finally the counting formula for the paired spin-singlet states is obtained in section 0 , filling in some of the details of the discussion in 10 . In section 8, counting formulas for the RR states at general level $k$ are obtained, while section 9 deals with the counting formulas for the general $k$ NASS states. Section 10 is devoted to discussions. In this paper, we do not go into the details of the numerical analysis, but refer to the papers [12, 6, 9, 10]. For all the cases checked, the results of the numerical diagonalization studies are exactly reproduced by the counting formulas.

\section{Construction of clustered quantum Hall states}

Clustered quantum Hall states are constructed as correlators in certain CFTs. But before we come to this construction, let us first explain the nomenclature 'clustered states'. Clustered states (with order $k$ clustering) have the following form

$$
\Psi_{k}^{M}\left(\left\{z_{i}\right\}\right)=\Phi_{\text {bos }}^{k}\left(\left\{z_{i}\right\}\right) \prod_{i<j}\left(z_{i}-z_{j}\right)^{M}
$$

where $\Phi_{\text {bos }}^{k}$ is a bosonic factor (symmetric under the exchange of any two coordinates) with the property

$$
\begin{cases}\Phi_{\mathrm{bos}}^{k}\left(z_{1}=z_{2}=\cdots=z_{i}\right) \neq 0 & i \leq k \\ \Phi_{\mathrm{bos}}^{k}\left(z_{1}=z_{2}=\cdots=z_{i}\right)=0 & i>k,\end{cases}
$$

i.e. the wave function vanishes as soon as $k+1$ or more particles are at the same location. Note that we omitted the exponential factors of the wave functions. The factor $\prod_{i<j}\left(z_{i}-z_{j}\right)^{M}$ is the familiar Laughlin factor. The fermionic quantum Hall states all have $M$ odd, while the bosonic versions would have $M$ even. Though changing $M$ changes many properties of the states, such as the filling fraction, the quasihole charge and statistics, the quasihole degeneracies are unaffected, and the counting formulas presented in this paper hold for any $M$. Note that this degeneracy is not to be confused with the 'torus degeneracy', which in fact does depend on $M$.

Following the pioneering paper [2], quantum Hall states can, under certain restrictions, be defined as correlators in CFTs. For the clustered quantum Hall states, CFTs with affine Lie algebra symmetry are used. The operators creating the electrons in general consist of a parafermion field 
and a vertex operator of a set of $r$ chiral boson fields ( $r$ is the rank of the affine Lie algebra). These boson fields take care of the charge, spin and possibly other quantum numbers associated to the particles. The electron creation operators take the form

$$
V_{\mathrm{el}}\left(z_{i}\right)=\psi_{\alpha}\left(z_{i}\right): e^{i \boldsymbol{\beta} \cdot \boldsymbol{\varphi}}\left(z_{i}\right):
$$

where $\psi_{\alpha}$ is a parafermion field and $\varphi$ is a set of free boson fields. The quantum Hall states can be now be defined as follows

$$
\Psi\left(\left\{z_{i}\right\}\right)=\lim _{z_{\infty} \rightarrow \infty} z_{\infty}^{a}\left\langle V_{\mathrm{el}, 1} V_{\mathrm{el}, 2} \cdots V_{\mathrm{el}, N}: e^{-i \mathbf{b} \cdot \boldsymbol{\varphi}}\left(z_{\infty}\right):\right\rangle
$$

The parameter $\mathbf{b}$ in the background charge needs to be chosen in such a way that the overall correlator is charge neutral. $a$ is chosen such that the effect of the background charge does not go to zero in the limit $z_{\infty} \rightarrow \infty$, whilst keeping the result finite. Moreover, the (unique) fusion of the parafermion fields $\psi$ must result in the identity operator $\mathbf{1}$ in order to get a non-zero correlator. This in general puts a restriction on the number of electrons $N$. In this paper, we will not give explicit results for the correlators (and thereby the wave functions $\Psi_{k}^{M}$ ). These wave functions and other properties of the ground state, such as the filling fraction, can be found in [6] for the Read-Rezayi states and in [10] for the spin-singlet analogues proposed in [7]. A different form of the Read-Rezayi wave functions can be found in [13]. Also, wave functions for states with quasiholes (see below) are presented there.

States with excitations (quasiholes) present are also defined in terms of correlators in the same CFT as the parent state, by inserting the corresponding quasihole operator in the correlator. Certain properties of these quasiholes can be studied via the corresponding correlators. A constraint on these operators follows from the condition that the wave function has to be analytical in the electron coordinates (the lowest Landau level condition). This implies that the quasihole operators need to be local with respect to the electron operators. This constrains the quasihole operators to be of the following form

$$
V_{\mathrm{qp}}(w)=\sigma_{\varpi}: e^{i \boldsymbol{\beta}^{\prime} \cdot \boldsymbol{\varphi}}(w):,
$$

where $\sigma_{\varpi}$ is a 'spin field' of the parafermion CFT. Note that the nomenclature spin field does not refer to the electron spin. Again, one has to insert a background charge to enforce charge neutrality. Also, upon fusing the parafermion and spin fields, one has to fuse to the identity operator 1 in the last step, to obtain a non-zero correlator. This constrains the possible particles in the correlator. The fusion of the spin fields is not unique: in general there is more than one fusion channel. This implies that a correlator with several quasihole operators in general stands for more than one quantum Hall state. In other words, the clustered quantum Hall states with quasiholes are degenerate. It is this degeneracy which lies at the heart of the non-abelian statistics.

One immediate question one can ask is how many states does one describe with such correlators? In fact, this question can be answered in two independent ways. The first one is via a numerical diagonalization study of interacting electrons on the sphere, in the presence of a magnetic field. In this paper, we will follow the second approach, which is analytical, and uses the conformal field theory of the underlying parafermions. But before we come to this point, we have to spend some words on the numerical approach as well, in order to be able to adapt the analytical approach to the numerical setup. This is the subject of the next section; for a more details, we refer to [10].

\section{The SETUP OF THE NUMERICAL STUdIES}

Though we will not describe numerical diagonalization studies in depth in this paper, it is necessary to point out briefly in which setup they are done, because we need to adapt our calculations to be able to compare results. The numerical diagonalization is most easily done on the sphere. The interaction between the electrons is chosen such that the clustered state under investigation is 
the unique ground state (in the absence of quasihole excitations). Note that this interaction is an ultra local, many-body interaction, rather different from the long range Coulomb interaction. To 'tune' to the right filling fraction, a specific number of flux quanta need to penetrate the sphere. States with quasiholes can be studied by increasing the number of flux quanta (but keeping all the other parameters the same); this results in the creation of quasiholes, as can be seen from the Laughlin gauge argument. The number of flux quanta needed for a state on the sphere with quasiholes is given by

$$
N_{\phi}=\frac{1}{\nu} N-\mathcal{S}+\Delta N_{\phi}
$$

where $N$ is the total number of electrons, and $\Delta N_{\phi}$ the number of excess flux quanta, needed for the creation of the quasiholes. $\mathcal{S}$ is an integer constant depending on the state under investigation. Also, the number of quasiholes which are created by increasing the number of flux quanta by one depends on the state under investigation. For the spin-polarized RR states, this relation is given by $n=k \Delta N_{\phi}$, where $n$ is the number of quasiholes. For the spin-singlet analogues, we have $n=n_{\uparrow}+n_{\downarrow}=2 k \Delta N_{\phi}$.

For the clustered quantum Hall states with quasiholes present, the ground state is degenerate (for the ultra local interaction). The degeneracy consists of two parts. First of all, there is an orbital degeneracy, which is caused by the fact that in this setup, the quasiholes are non-local. This orbital degeneracy is not specific for clustered states; it is also present for the (unpaired) Laughlin states. For a system in which the quasiholes are localized, this degeneracy would not be present. Secondly, there is an intrinsic degeneracy, which stems from the non-trivial fusion rules of the spin fields, needed to create quasihole excitations. This source of degeneracy is special for the clustered states. In this paper, we will focus on this intrinsic degeneracy and obtain analytical expressions, which allow the combination with the orbital degeneracy factors. This provides us with explicit expressions for the degeneracy of the ground states, in the presence of quasiholes.

As spin and angular momentum are good quantum numbers, all the states obtained from the numerical diagonalization fall into spin and angular momentum multiplets. The structure of the counting formulas is such that also the multiplet structure can be extracted.

\section{Degeneracy factors and Counting Formulas}

The intrinsic degeneracy is caused by the non-trivial fusion rules of the spin fields. As an example, we will use the spin fields of the $\mathfrak{s u}(3)_{2} / \mathfrak{u}(1)^{2}$ parafermionic CFT. The fields and their fusion rules in this theory can be determined according to the methods of [11] and are summarized in table 1. We use the notation introduced in [10]. The parafermion fields are denoted by $\psi$, and all have conformal dimension $\Delta_{\psi}=\frac{1}{2}$. In particular, $\psi_{1}, \psi_{2}, \psi_{12}$ correspond to the roots $\alpha_{1},-\alpha_{2}$ and $\alpha_{1}+\alpha_{2}$ of $s u(3)$, respectively. The spin fields $\sigma_{\uparrow}, \sigma_{\downarrow}, \sigma_{3}$ and $\rho$ are related to the weights of $s u(3)$ and their conformal dimensions are given by $\Delta_{\sigma}=\frac{1}{10}$ and $\Delta_{\rho}=\frac{3}{5}$. The fusion of an arbitrary number of $\sigma_{\uparrow, \downarrow}$ fields can be depicted in a Bratteli diagram (see also [10]). Each arrow in the

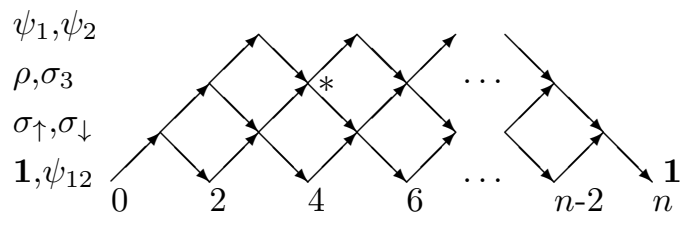

FiguRE 1. Bratteli diagram for the spin fields of $\mathfrak{s u}(3)_{2} / \mathfrak{u}(1)^{2}$.

diagram in figure 1 stands for either a $\sigma_{\uparrow}$ or $\sigma_{\downarrow}$ field. The arrow starts at a certain field which 


\begin{tabular}{c|c|c|c|c|c|c|c}
$\times$ & $\sigma_{\uparrow}$ & $\sigma_{\downarrow}$ & $\sigma_{3}$ & $\rho$ & $\psi_{1}$ & $\psi_{2}$ & $\psi_{12}$ \\
\hline$\sigma_{\uparrow}$ & $\mathbf{1}+\rho$ & & & & & & \\
$\sigma_{\downarrow}$ & $\psi_{12}+\sigma_{3}$ & $\mathbf{1}+\rho$ & & & & & \\
$\sigma_{3}$ & $\psi_{1}+\sigma_{\downarrow}$ & $\psi_{2}+\sigma_{\uparrow}$ & $\mathbf{1}+\rho$ & & & & \\
$\rho$ & $\psi_{2}+\sigma_{\uparrow}$ & $\psi_{1}+\sigma_{\downarrow}$ & $\psi_{12}+\sigma_{3}$ & $\mathbf{1}+\rho$ & & & \\
$\psi_{1}$ & $\sigma_{3}$ & $\rho$ & $\sigma_{\uparrow}$ & $\sigma_{\downarrow}$ & $\mathbf{1}$ & & \\
$\psi_{2}$ & $\rho$ & $\sigma_{3}$ & $\sigma_{\downarrow}$ & $\sigma_{\uparrow}$ & $\psi_{12}$ & $\mathbf{1}$ & \\
$\psi_{12}$ & $\sigma_{\downarrow}$ & $\sigma_{\uparrow}$ & $\rho$ & $\sigma_{3}$ & $\psi_{2}$ & $\psi_{1}$ & $\mathbf{1}$
\end{tabular}

TABLE 1. Fusion rules of the parafermion and spin fields associated to the parafermion theory $\mathfrak{s u}(3)_{2} / \mathfrak{u}(1)^{2}$ introduced by Gepner [11.

can only be one of the fields on the left of the diagram at the same height. This last field is fused with the one corresponding to the arrow, while the arrow points at a field present in this fusion. As an example, the arrows starting at the $*$ are encoding the fusion rules $\rho \times \sigma_{\uparrow(\downarrow)}=\psi_{2(1)}+\sigma_{\uparrow(\downarrow)}$ and $\sigma_{3} \times \sigma_{\uparrow(\downarrow)}=\psi_{1(2)}+\sigma_{\downarrow(\uparrow)}$. One checks that the diagram is in accordance with the first two columns of Table 1 .

From figure 1, one immediately reads off that in general there is more than one fusion path of spin fields with leads to the identity (possibly the identity is reached only after the fusion with the parafermion fields $\psi_{1,2}$ of the electron operators). It is easily seen that the number of fusion channels starting from and terminating at $\mathbf{1}$ while $n_{\uparrow} \sigma_{\uparrow}$ and $n_{\downarrow} \sigma_{\downarrow}$ spin fields are fused is given by

$$
d_{n_{\uparrow}, n_{\downarrow}}=\mathcal{F}\left(n_{\uparrow}+n_{\downarrow}-2\right),
$$

where $\mathcal{F}(n)$ is the $n$-th Fibonacci number, defined by $\mathcal{F}(n)=\mathcal{F}(n-1)+\mathcal{F}(n-2)$ with the initial conditions $\mathcal{F}(0)=1$ and $\mathcal{F}(1)=1$. Next to this intrinsic degeneracy, there is an orbital degeneracy. These orbital degeneracy factors can be found in 19, 10 for the states discussed in this paper. These factors have the general form

$$
\prod_{i}\left(\begin{array}{c}
\frac{n_{i}-F_{i}}{k}+n_{i} \\
n_{i}
\end{array}\right)
$$

The product is over the types of quasiholes, while the numbers $F_{i}$ are interpreted as the number of 'unclustered' particles in the state. In the correlators, these correspond to the fundamental parafermions $\psi_{i}$. For each fusion path, these numbers can be different, implying that we have to split the intrinsic degeneracy according to these numbers. We denote these 'split degeneracy factors' by \{\}$_{k}$. Explicitly, we have $\left\{\begin{array}{l}n \\ F\end{array}\right\}_{k}$ and $\left\{\begin{array}{l}n_{\uparrow} n_{\downarrow} \\ F_{1} F_{2}\end{array}\right\}_{k}$ for the RR and NASS states, respectively.

Using the notation above, the counting formula for the clustered spin-singlet quantum Hall states take the following form

$$
\#_{\mathrm{NASS}}\left(N, \Delta N_{\phi}, k\right)=\sum_{N_{\uparrow, \downarrow}, n_{\uparrow, \downarrow}, F_{1,2}}^{\prime}\left\{\begin{array}{ll}
n_{\uparrow} & n_{\downarrow} \\
F_{1} & F_{2}
\end{array}\right\}_{k}\left(\begin{array}{c}
\frac{N_{\uparrow}-F_{1}}{k}+n_{\uparrow} \\
n_{\uparrow}
\end{array}\right)\left(\begin{array}{c}
\frac{N_{\downarrow}-F_{2}}{k}+n_{\downarrow} \\
n_{\downarrow}
\end{array}\right),
$$

where the prime on the summation indicates the presence of constraints (see below eq. (51)). The equivalent counting formula for the Read-Rezayi states is given in eq. (43).

The new result of this paper are the explicit split degeneracy factors for the $\mathbb{Z}_{k}$ and $\mathfrak{s u}(3)_{k} / \mathfrak{u}(1)^{2}$ parafermions at level $k>2$ (for $k=2$, these factors can be found in [12] and [10] respectively). Previously, these factors for the $\mathbb{Z}_{k}$ parafermions $(k>2)$ could only be characterized via recursion relations, see [9, 14]. Note that the results in this paper are easily extended to the more general $\mathfrak{s u}(N)_{k} / \mathfrak{u}(1)^{N-1}$ parafermions. 
We will now briefly outline in which way the split degeneracy factors are obtained. The starting point is the character of the parafermionic CFT. The symbols \{\}$_{k}$ can be extracted from finitized forms of these characters [9] (see also [15]). Recursion relations for these finitized characters can be derived from an explicit basis of the parafermionic CFT. These recursion relations will be written in a way that allows for an explicit solution, from which the symbols \{\}$_{k}$ can be extracted. In the sections 58, we will demonstrate this for the level $k=2$ spin-singlet states of [7].

\section{A BASIS FOR THE $\mathfrak{s u}(3)_{2} / \mathfrak{u}(1)^{2}$ PARAFERMION THEORY}

In this section, we briefly describe how an explicit basis for the chiral spectrum of the $\mathfrak{s u}(3)_{2} / \mathfrak{u}(1)^{2}$ parafermion CFT is formed. The starting point is the chiral character for the parafermions in the $\mathfrak{s u}(3)_{2} / \mathfrak{u}(1)^{2}$ conformal field theory. This character can be written in the form of a 'Universal Chiral Partition Function' (UCPF) see, for instance, [16, 17]. This character reads [18]

$$
\operatorname{ch}\left(x_{1}, x_{2} ; q, k=2\right)=\sum_{n_{1}, n_{2}} \frac{q^{\left(n_{1}^{2}+n_{2}^{2}-n_{1} n_{2}\right) / 2}}{(q)_{n_{1}}(q)_{n_{2}}} x_{1}^{n_{1}} x_{2}^{n_{2}} .
$$

In this character, $x_{i}=e^{\beta \mu_{i}}$ are fugacities of the particles, and $q=e^{\beta \varepsilon}$ ( $\beta$ is the inverse temperature). $(q)_{a}$ is defined by $(q)_{a}=\prod_{k=1}^{a}\left(1-q^{k}\right)$ for $a>0$ and $(q)_{0}=1$.

The bilinear form in the exponent of $q$ is described by the matrix

$$
\mathbb{K}=\left(\begin{array}{cc}
1 & -\frac{1}{2} \\
-\frac{1}{2} & 1
\end{array}\right)
$$

The same matrix also describes the exclusion statistics of these parafermions. More information on the relation between exclusion statistics and the UCPF can be found in [19].

A basis for a CFT can be thought of as a set of states spanning the chiral Hilbert space. This set of states can be written as a (set of) vacuum state(s), on which creation operators act. The parafermions $\psi_{1,2}(z)$ in the $\mathfrak{s u}(3)_{2} / \mathfrak{u}(1)^{2}$ theory can be expanded in modes as

$$
\psi_{1,2}(z)=\sum_{m \in \mathbb{Z}} z^{-m} \psi_{m-\frac{1}{2}}^{1,2} .
$$

As usual, the modes $\psi_{m}$ with negative index are the creation operators while the modes with positive index annihilate the vacuum

$$
\psi_{m}|0\rangle=0 \quad m>0
$$

The set of states

$$
\psi_{-s_{n}}^{a_{n}} \psi_{-s_{n-1}}^{a_{n-1}} \cdots \psi_{-s_{1}}^{a_{1}}|0\rangle
$$

is overcomplete, because of the (generalized) commutation rules of the parafermions. In the following, we will point out which restrictions on the indices $s_{i}$ will remove the 'overcompleteness'. In doing so, we will follow the exclusion interpretation of the K-matrix as closely as possible and concentrate on the lowest possible 'energy' (given by $L_{0}=\sum_{i} s_{i}$ ) for a certain number of applied fields first. The ordering of the modes $\psi^{1,2}$ is such that we apply the $\psi^{1}$ modes first. From (12) it follows that the simplest non-trivial state is

$$
\psi_{-1 / 2}^{1}|0\rangle \text {. }
$$

Interpreting the matrix (11) as the exclusion statistics matrix, the minimal spacing between two $\psi^{1}$ modes is 1 , thus the state with two $\psi^{1}$ 's acting on the vacuum with minimal energy is

$$
\psi_{-3 / 2}^{1} \psi_{-1 / 2}^{1}|0\rangle \text {. }
$$


The extension to $n_{1} \psi^{1}$ modes is simple

$$
\psi_{-\left(2 n_{1}-1\right) / 2}^{1} \cdots \psi_{-3 / 2}^{1} \psi_{-1 / 2}^{1}|0\rangle .
$$

Note that if this were the whole story, we would describe the (free) Majorana fermion. The spacing between $\psi^{2}$ modes is the same as for the $\psi^{1}$ modes. However, if one acts with $\psi^{2}$ on a state in which $\psi^{1}$ modes are already present, one has to take into account the mutual statistics between $\psi^{1}$ and $\psi^{2}$ modes, which is $-1 / 2$, according to (11). Thus the energies of the $\psi^{2}$ modes have an extra shift of $-n_{1} / 2$, resulting in the following states (with minimal energy)

$$
\psi_{-\left(2 n_{2}-1-n_{1}\right) / 2}^{2} \cdots \psi_{-\left(3-n_{1}\right) / 2}^{2} \psi_{-\left(1-n_{1}\right) / 2}^{2} \psi_{-\left(2 n_{1}-1\right) / 2}^{1} \cdots \psi_{-3 / 2}^{1} \psi_{-1 / 2}^{1}|0\rangle .
$$

The (dimensionless) energy associated to this state is $\frac{n_{1}^{2}+n_{2}^{2}-n_{1} n_{2}}{2}$, precisely the exponent of $q$ in the character (10). To obtain all the possible states, one has to allow states with higher energies as well. As usual [14], the energies of all the modes can have integer shifts, under the restriction that modes acting on a state have larger energies than the modes of the same type which have been applied earlier. This results in the following set of states

$$
\psi_{-\left(2 n_{2}-1-n_{1}\right) / 2-t_{n_{2}}}^{2} \cdots \psi_{-\left(3-n_{1}\right) / 2-t_{2}}^{2} \psi_{-\left(1-n_{1}\right) / 2-t_{1}}^{2} \psi_{-\left(2 n_{1}-1\right) / 2-s_{n_{1}}}^{1} \cdots \psi_{-3 / 2-s_{2}}^{1} \psi_{-1 / 2-s_{1}}^{1}|0\rangle,
$$

with $s_{n_{1}} \geq \ldots \geq s_{2} \geq s_{1} \geq 0$ and $t_{n_{2}} \geq \ldots \geq t_{2} \geq t_{1} \geq 0\left(s_{i}, t_{j} \in \mathbb{N}\right)$.

Up to now, we used the special ordering of applying modes to the vacuum, namely, all the $\psi^{1}$ modes first. This is in fact enough to span the whole chiral spectrum, as can be seen if we perform the trace over all basis states. More or less by construction, we obtain the character (10). However, we also can allow a general ordering of the modes. As an example, we take the following state

$$
\psi_{-0}^{2} \psi_{-1 / 2}^{1}|0\rangle \text {. }
$$

The energy of the $\psi^{2}$ mode is zero because it gets an extra shift of $-1 / 2$ due to the presence of the $\psi^{1}$ mode. In spanning the whole chiral spectrum, we can also choose to use the state, with the order of the modes changed

$$
\psi_{-0}^{1} \psi_{-1 / 2}^{2}|0\rangle
$$

In this case, the $\psi^{1}$ mode gets an extra shift of $-1 / 2$, because of the presence of the $\psi^{2}$ mode. Thus, the $L_{0}$ value is the same for both states. In general, changing the order of two neighbouring $\psi^{1}$ and $\psi^{2}$ modes does not change the $L_{0}$ value if the extra shifts are changed in the appropriate way. The extra shift of a field is given by $-1 / 2$ times the number of preceding modes of the other type. In general, two states related by a reordering of modes are different, but we can use either of them (but not both) to span the chiral spectrum. Note that the rules of the spacing between the various fields is in accordance with the (exclusion) statistics interpretation of the matrix $\mathbb{K}$. The character (10) is obtained by taking the trace over all the states in the basis (19)

$$
\operatorname{ch}\left(x_{1}, x_{2} ; q\right)=\operatorname{Tr} x_{1}^{n_{1}} x_{2}^{n_{2}} q^{L_{0}} .
$$

We can now define the finitized characters needed in the derivation of the symbols \{\}$_{k}$ by using the basis described above. These finitized characters are polynomials which will be denoted by $Y_{(l, m)}$. These polynomials are traces over the basis (19), but restricted to the states in which the energy of the modes of the $\psi_{1}\left(\psi_{2}\right)$ fields are smaller or equal to $l(m)$. Though the total energy of a state does not depend on the ordering of the modes, the energies of the individual modes do depend on the ordering, as can be seen by comparing the states (20) and (21). By restricting the trace over states in which the labels of the modes are bounded, we must include a state if there is 
at least one ordering in which all the modes satisfy the bounds imposed. Note that there may be other orderings, in which these bounds are not satisfied. We write the finitized characters as

$$
Y_{(l, m)}\left(x_{1}, x_{2} ; q\right)=\operatorname{Tr}_{\leq l, \leq m}^{\prime} x_{1}^{n_{1}} x_{2}^{n_{2}} q^{L_{0}} .
$$

The prime on the trace denotes an important restriction on the number of modes (denoted by $n_{1}$ and $\left.n_{2}\right)$ present in the states. These numbers must satisfy $n_{1}=2 l(\bmod 2)$ and $n_{2}=2 m(\bmod 2)$. This restriction takes into account that after fusing the spin fields, one ends up in the right sector, which can be $\mathbf{1}, \psi_{1}, \psi_{2}$ or $\psi_{12}$ depending on the number of spin-up and down electrons. This is necessary, because after fusing the spin fields and the parafermion fields of the electron operators, on has to end with the identity $\mathbf{1}$, to obtain a non-zero correlator.

The finitized characters $Y_{(l, m)}$ can be written in terms of recursion relations of the following form

$$
\begin{aligned}
& Y_{(l, m)}=Y_{(l-1, m)}+x_{1} q^{l-\frac{1}{2}} Y_{\left(l-1, m+\frac{1}{2}\right)} \\
& Y_{(l, m)}=Y_{(l, m-1)}+x_{2} q^{m-\frac{1}{2}} Y_{\left(l+\frac{1}{2}, m-1\right)} .
\end{aligned}
$$

Note that the recursion relations above are stated in terms of the energy labels of the modes. The aim we have is finding the number of possible states when a certain number of extra flux is added. We therefore need to make a change to labels which depend on the additional flux. In fact, we will use the number of particles (given by $n_{\uparrow}$ and $n_{\downarrow}$ in this case) created by this flux as labels for the

finitized partition functions. Explicitly, we have $l=\frac{n_{\uparrow}}{2}$ and $m=\frac{n_{\downarrow}}{2}$. In terms of the number of created quasiholes, the recursion relations become (compare [10])

$$
\begin{aligned}
& Y_{\left(n_{\uparrow}, n_{\downarrow}\right)}=Y_{\left(n_{\uparrow}-2, n_{\downarrow}\right)}+x_{1} q^{\frac{n_{\uparrow}-1}{2}} Y_{\left(n_{\uparrow}-2, n_{\downarrow}+1\right)}, \\
& Y_{\left(n_{\uparrow}, n_{\downarrow}\right)}=Y_{\left(n_{\uparrow}, n_{\downarrow}-2\right)}+x_{2} q^{\frac{n_{\downarrow}-1}{2}} Y_{\left(n_{\uparrow}+1, n_{\downarrow}-2\right)} .
\end{aligned}
$$

The initial conditions for these recursion relations look as follows

$$
\begin{aligned}
& Y_{(1,0)}=Y_{(0,1)}=0, \\
& Y_{(0,0)}=Y_{(2,0)}=Y_{(0,2)}=1, \\
& Y_{(1,1)}=q^{\frac{1}{2}} x_{1} x_{2} .
\end{aligned}
$$

The finitized characters are completely described by (25) and (26). In the next section, we will solve these recursion relations and thereby provide explicit expressions for the finitized characters.

\section{RECURSION RELATIONS AND SOLUTIONS}

The recursion relations of the previous section can be solved explicitly; we will follow the approach of [20]. The key observation is that the recursion relations can be matched to general recursion relations, which are solved in terms of finitizations of universal chiral partition functions. Consider the following polynomials $P$

$$
P_{\mathbf{L}}(\mathbf{z} ; q)=\sum_{\mathbf{m}}\left(\prod_{i} z_{i}^{m_{i}}\right) q^{\frac{1}{2} \mathbf{m} \cdot \mathbb{K} \cdot \mathbf{m}+\mathbf{Q} \cdot \mathbf{m}} \prod_{i}\left[\begin{array}{c}
(\mathbf{L}+(\mathbb{I}-\mathbb{K}) \cdot \mathbf{m}+\mathbf{u})_{i} \\
m_{i}
\end{array}\right] .
$$

In this equation, $\mathbb{I}$ is the identity matrix, $\mathbb{K}$ the statistics matrix and $\left[\begin{array}{l}a \\ b\end{array}\right]$ the $q$-deformed binomial ( $q$-binomial)

$$
\left[\begin{array}{l}
a \\
b
\end{array}\right]=\left\{\begin{array}{cc}
\frac{(q)_{a}}{(q)_{b}(q)_{a-b}} & a, b \in \mathbb{N} ; b \leq a \\
0 & \text { otherwise }
\end{array} .\right.
$$


Note that we defined the $q$-binomial to be non-zero only if both entries are integers greater or equal to zero, to avoid additional constraints on the sums in the counting formulas.

From the definition of the $q$-binomials, the following identity is easily derived

$$
\left[\begin{array}{l}
a \\
b
\end{array}\right]=\left[\begin{array}{c}
a-1 \\
b
\end{array}\right]+q^{a-b}\left[\begin{array}{l}
a-1 \\
b-1
\end{array}\right] .
$$

Replacing the $i$ 'th $q$-binomial factor in (27) by the right hand side of (29), one finds the following recursion relations

$$
P_{\mathbf{L}}(\mathbf{z} ; q)=P_{\mathbf{L}-\mathbf{e}_{i}}(\mathbf{z} ; q)+z_{i} q^{-\frac{1}{2} \mathbb{K}_{i i}+\mathbf{Q}_{i}+\mathbf{u}_{i}+\mathbf{L}_{i}} P_{\mathbf{L}-\mathbb{K} \cdot \mathbf{e}_{i}}(\mathbf{z} ; q) .
$$

The vector $\mathbf{e}_{i}$ represents a unit vector in the $i$ th direction. We will use the equivalence between (27) and (30) frequently, because the recursion relations we encounter in deriving the counting formulas are all of type (30). Of course, upon deriving polynomials from recursion relations, one has to take the initial conditions into account. For the counting we need to know the finitizations of the character formulas, and these can be written in the form (27). Thus, when we solve recursion relations by polynomials of the form (27), the proper initial conditions are automatically taken into account.

We start by applying the above to the recursion relations (24), resulting in the following expressions for the truncated characters $Y_{\left(n_{\uparrow}, n_{\downarrow}\right)}$

$$
Y_{\left(n_{\uparrow}, n_{\downarrow}\right)}\left(x_{1}, x_{2} ; q\right)=\sum_{a, b} q^{\left(a^{2}+b^{2}-a b\right) / 2} x_{1}^{a} x_{2}^{b}\left[\begin{array}{c}
\frac{n_{\uparrow}+b}{2} \\
a
\end{array}\right]\left[\begin{array}{c}
\frac{n_{\downarrow}+a}{2} \\
b
\end{array}\right] .
$$

This result will be needed for the final counting formula, which we give in the next section.

\section{A COUnting Formula for the NASS State AT $k=2$}

From the truncated characters of the previous section, we can obtain the symbols \{\}$_{2}$, needed in the counting formula eq. (9). In fact, the symbols \{\}$_{2}$ are obtained by taking the limit $q \rightarrow 1$ of the coefficient of $x_{1}^{F_{1}} x_{2}^{F_{2}}$ in eq. (31) (see, for instance, [9, 10])

$$
Y_{\left(n_{\uparrow}, n_{\downarrow}\right)}\left(x_{1}, x_{2} ; 1\right)=\sum_{F_{1}, F_{2}} x_{1}^{F_{1}} x_{2}^{F_{2}}\left\{\begin{array}{ll}
n_{\uparrow} & n_{\downarrow} \\
F_{1} & F_{2}
\end{array}\right\} .
$$

In this limit, the $q$-binomials in (31) become 'ordinary' binomials and we find

$$
\left\{\begin{array}{cc}
n_{\uparrow} & n_{\downarrow} \\
F_{1} & F_{2}
\end{array}\right\}_{2}=\left(\begin{array}{c}
\frac{n_{\uparrow}+F_{2}}{2} \\
F_{1}
\end{array}\right)\left(\begin{array}{c}
\frac{n_{\downarrow}+F_{1}}{2} \\
F_{2}
\end{array}\right) .
$$

The fact that the finitized characters indeed provide the symbols \{\} is rather non-trivial. This connection was first proposed in [9]. Some (restricted) 'solid on solid' (SOS) models (see, for instance [21]) can be mapped to the Bratteli diagrams of the spin fields of the quasiholes. Recursion relations for the partition functions for these models (at finite size) are in general equivalent to recursion relations for finitized characters in certain CFTs. In the case at hand, the corresponding CFT is the parafermion CFT. This provides a link between the Bratteli diagrams and the parafermion theories. As a check, on can calculate the number of fusion paths for the spin fields by summing over the symbols \{\} and compare to the result obtained from the diagram itself. In this specific case, the equivalence follows from the structure of the recursion relations (see for instance [10]), giving rise to the identity

$$
\sum_{F_{1}, F_{2}}^{\prime}\left(\begin{array}{c}
\frac{n_{\uparrow}+F_{2}}{2} \\
F_{1}
\end{array}\right)\left(\begin{array}{c}
\frac{n_{\downarrow}+F_{1}}{2} \\
F_{2}
\end{array}\right)=\mathcal{F}\left(n_{\uparrow}+n_{\downarrow}-2\right) .
$$


The prime on the summation denotes the constraints $F_{1} \equiv n_{\downarrow}(\bmod 2)$ and $F_{2} \equiv n_{\uparrow}(\bmod 2)$. At the level of the wave functions, the degeneracy is due to the presence of particles which do not belong to a cluster any more. At the level of correlators, these unclustered particles correspond to parafermions $\psi^{1}$ and $\psi^{2}$, which act as 'cluster breakers'. In the case of the pfaffian state, this was made explicit in $\mid 12$.

The counting formula for the NASS state at $k=2$ is obtained by inserting the symbol (33) in the general counting formula (9)

$$
\#\left(N, \Delta N_{\phi}, k=2\right)=\sum_{N_{\uparrow, \downarrow}, n_{\uparrow, \downarrow}, F_{1,2}}^{\prime}\left(\begin{array}{c}
\frac{n_{\uparrow}+F_{2}}{2} \\
F_{1}
\end{array}\right)\left(\begin{array}{c}
\frac{n_{\downarrow}+F_{1}}{2} \\
F_{2}
\end{array}\right)\left(\begin{array}{c}
\frac{N_{\uparrow}-F_{1}}{2}+n_{\uparrow} \\
n_{\uparrow}
\end{array}\right)\left(\begin{array}{c}
\frac{N_{\downarrow}-F_{2}}{2}+n_{\downarrow} \\
n_{\downarrow}
\end{array}\right),
$$

where the prime on the sum indicates the constraints $N_{\uparrow}+N_{\downarrow}=N, n_{\uparrow}+n_{\downarrow}=4 \Delta N_{\phi}$ and $N_{\uparrow}-N_{\downarrow}=n_{\downarrow}-n_{\uparrow}$.

We will now comment on the spin and angular momentum multiplet structure. As an example, we will write out the polynomial $Y_{(7,1)}$

$$
Y_{(7,1)}=\left(q^{\frac{1}{2}}+q^{\frac{3}{2}}+q^{\frac{5}{2}}+q^{\frac{7}{2}}\right) x_{1} x_{2}+\left(q^{\frac{7}{2}}+2 q^{\frac{9}{2}}+2 q^{\frac{11}{2}}+2 q^{\frac{13}{2}}+q^{\frac{15}{2}}\right) x_{1}^{3} x_{2}+q^{\frac{19}{2}} x_{1}^{5} x_{2}^{3} .
$$

After multiplying the coefficient of $x_{1}^{F_{1}} x_{2}^{F_{2}}$ with (in general) $q^{-\left(n_{\uparrow} F_{1}+n_{\downarrow} F_{2}\right) / 4}$, one obtains a sum of terms of the form $q^{l_{z}}$, which together form a collection of angular momentum multiplets with quantum numbers $l_{z}$. For instance, the coefficient of $x_{1}^{3} x_{2}$ in eq. (36) encodes two multiplets, namely $L=2$ and $L=1$.

An alternative way to obtain these results is by associating angular momentum multiplets to the binomials in eq. (35). The binomials $\left(\begin{array}{c}a \\ f\end{array}\right)$ forming the symbols \{\}$_{2}$ need to be interpreted as the number of ways on can put $f$ fermions in $a$ boxes, which are labeled with $l_{z}=-\frac{(a-1)}{2},-\frac{(a-1)}{2}+$

$1, \ldots, \frac{(a-1)}{2}$ angular momentum quantum numbers. Each way of putting the $f$ fermions in $a$ boxes has an $l_{z}^{\text {tot }}$ associated with it. Together, these $l_{z}^{\text {tot }}$ quantum numbers fall into angular momentum multiplets. In this way, angular momentum multiplets can be associated with the binomials. The angular momentum multiplets of the various binomials in the counting formula need to be added in the usual way.

Though the parafermion theory does not have a proper $S U(2)$ spin symmetry, one can associate spin quantum numbers to every state by taking $S_{z}=\frac{N_{\uparrow}-N_{\downarrow}}{2}$. Combining the spin and angular momentum, one finds that all the states fall into spin and angular momentum multiplets.

The numerical diagonalization studies for the NASS state at level $k=2$ are described in [10]. It is very gratifying to see that the counting formula eq. (35) does in fact exactly reproduce the quasihole degeneracies, as well as the multiplet structure.

In order to find the counting results for the spin-singlet states at general level- $k$, we first take a closer look at the counting of the Read-Rezayi states, which was in fact done in [9]. Those results however, were stated in terms of recursion relations which are difficult to solve. The advantage of the recursion relations presented in the next section is that they can easily be solved in terms of ( $q$-deformed) binomials, and thus provide explicit expressions for the symbols \{\}$_{k}$.

\section{Counting FORmulas For the Read-Rezayi states}

The derivation of the counting formulas for the RR states goes along the same lines as the derivation for the NASS $\mathrm{k}=2$ states as explained in the previous sections. Therefore, we do not go into full detail, but concentrate on the parts which need more explanation. 
We start with the character of the $\mathfrak{s u}(2)_{k} / \mathfrak{u}(1)$ parafermionic theory (see [22]), which can be obtained from 23, 18, 24]

$$
\operatorname{ch}(x ; q, k)=\sum_{a_{i}} \frac{q^{\frac{1}{2}\left(\mathbf{a} \cdot \mathbb{C}_{k-1} \cdot \mathbf{a}\right)}}{\prod_{i}(q)_{a_{i}}} x^{i a_{i}}
$$

where $\mathbf{a}=\left(a_{1}, \ldots, a_{k-1}\right)$ and $\mathbb{C}_{k-1}=2 \mathbb{A}_{k-1}^{-1}, \mathbb{A}_{k-1}$ being the Cartan matrix of $s u(k)$. In components, these matrices are given by

$$
\begin{aligned}
& \left(\mathbb{A}_{k-1}\right)_{i, j}=2 \delta_{i, j}-\delta_{|i-j|, 1} \\
& \left(\mathbb{A}_{k-1}^{-1}\right)_{i, j}=\min (i, j)-\frac{i j}{k} \quad i, j=1, \ldots, k-1 .
\end{aligned}
$$

In fact, $\mathbb{C}_{k-1}$ is the $\mathrm{K}$-matrix for the $\mathbb{Z}_{k}$ parafermions, see 17 . The parafermions in this theory are $\psi_{0}, \psi_{1}, \ldots \psi_{k-1}\left(\psi_{0}\right.$ is the identity $\mathbf{1}$ and the labels are defined modulo $k$ ). By writing $x^{i a_{i}}$ in the character (37), we take care of the fact that the fugacity of species $i$ is $i$ times the fugacity of the first type of particle. In fact, the $i$ 'th species can be thought of as a 'composite' of $i$ particles of species 1. This point of view is supported by the fusion rules for these parafermions $\psi_{1} \times \psi_{p}=\psi_{p+1}$, with $p=1, \ldots, k-1$. This structure is also present in the K-matrix structure describing the Read-Rezayi states [25, 26, 19].

A basis for the chiral spectrum can be constructed in the same way as described in section 5 . The shifts in modes between the various fields are given by the elements of the matrix $\mathbb{K}$. We will now proceed by directly giving the corresponding recursion relations

$$
Y_{\mathbf{l}}(x ; q, k)=Y_{\mathbf{l}-\mathbf{e}_{i}}+x^{i} q^{l_{i}-\frac{i(k-i)}{k}} Y_{\mathbf{l}-\mathbb{C}_{k-1} \cdot \mathbf{e}_{i}}
$$

The factor $\frac{i(k-i)}{k}$ is the conformal dimension of the $i$ 'th parafermion in the $\mathbb{Z}_{k}$-parafermion theory. These recursion relations are solved by the following polynomials

$$
Y_{\mathbf{l}}(x ; q, k)=\sum_{a_{i}} q^{\frac{1}{2}\left(\mathbf{a} \cdot \mathbb{C}_{\mathbf{k}-\mathbf{1}} \cdot \mathbf{a}\right)} \prod_{i=1}^{k-1} x^{i a_{i}}\left[\begin{array}{c}
\left(\mathbf{l}+\left(\mathbb{I}_{k-1}-\mathbb{C}_{k-1}\right) \cdot \mathbf{a}\right)_{i} \\
a_{i}
\end{array}\right],
$$

where $\mathbb{I}_{k-1}$ denotes the $(k-1)$-dimensional unit matrix. To obtain the counting results, we have to specify the truncation parameters $l_{i}$. As in the NASS case for $k=2$, we will do this in terms of the number of particles created by the extra flux, given by $n=k \Delta N_{\phi}$ for the states under consideration. Because the chemical potential of species $i$ is $i$ times the chemical potential of species 1 , the truncation parameter $l_{i}$ has to be scaled with a factor $i$ with respect to $l_{1}$ (see, for instance 19), which is found to be $l_{1}=\frac{n}{k}$. This leads to the following truncation parameters $l_{i}=\frac{i n}{k}$, and the truncated characters needed for the counting become

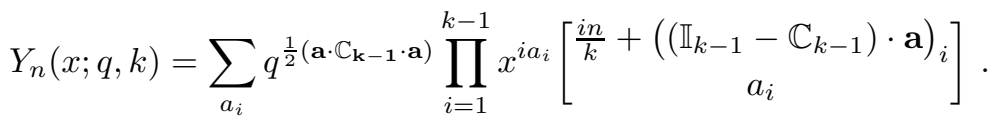

To obtain the symbols $\left\{\begin{array}{l}n \\ F\end{array}\right\}_{k}$ which are needed for the counting, one has to take the limit $q \rightarrow 1$ of the prefactor of $x^{F}$ in eq. (41). This results in

$$
\left\{\begin{array}{l}
n \\
F
\end{array}\right\}_{k}=\sum_{\sum i a_{i}=F} \prod_{i=1}^{k-1}\left(\begin{array}{c}
\frac{i n}{k}+\left(\left(\mathbb{I}_{k-1}-\mathbb{C}_{k-1}\right) \cdot \mathbf{a}\right)_{i} \\
a_{i}
\end{array}\right) .
$$


With this result, we arrive at the following counting formula for the Read-Rezayi states (for general $k$ )

$$
\#_{\mathrm{RR}}\left(N, \Delta N_{\phi}, k\right)=\sum_{F}\left\{\begin{array}{l}
n \\
F
\end{array}\right\}_{k}\left(\begin{array}{c}
\frac{N-F}{k}+n \\
n
\end{array}\right),
$$

with $n=k \Delta N_{\phi}$. To make the above (in particular the symbols \{\}$_{k}$ ) more explicit, we will discuss the $k=2$ (i.e. the pfaffian) and $k=3$ cases. For the pfaffian state counting, we need to know the symbols \{\}$_{2}$. Eq. (42) with $k=2$ gives $\left\{\begin{array}{c}n \\ F\end{array}\right\}_{2}=\left(\begin{array}{c}\frac{n}{2} \\ F\end{array}\right)$. Of course, this is just the result already found in [12]. Note that our notation is slightly different with respect to the one used in 12, 9]. In our notation, we denote the number of created quasiholes by $n$. In [12, 9], $n$ denoted the number of extra fluxes, which is denoted by $\Delta N_{\phi}$ in this paper.

Although the method described above seems to be unnecessarily complicated to reproduce this result, it is very useful for obtaining closed expressions for $k>2$. As an illustration, we will discuss the case $k=3$, and compare our results with [9]. For $k=3$, the polynomials are given by the following expression

$$
Y_{n}(x ; q, 3)=\sum_{a, b} q^{\frac{2}{3}\left(a^{2}+b^{2}+a b\right)} x^{a+2 b}\left[\begin{array}{c}
\frac{n}{3}-\frac{a+2 b}{3} \\
a
\end{array}\right]\left[\begin{array}{c}
\frac{2 n}{3}-\frac{2 a+b}{3} \\
b
\end{array}\right] .
$$

Indeed, these polynomials reduce to the ones in [9], upon setting $q=1$. The symbols \{\}$_{3}$ are now easily written down

$$
\left\{\begin{array}{l}
n \\
F
\end{array}\right\}_{3}=\sum_{a+2 b=F}\left(\begin{array}{c}
\frac{n}{3}-\frac{a+2 b}{3} \\
a
\end{array}\right)\left(\begin{array}{c}
\frac{2 n}{3}-\frac{2 a+b}{3} \\
b
\end{array}\right) .
$$

Note that only a finite number of terms contribute to the sum in eq. (45). In fact, this is true for all the symbols (42) with $n$ finite.

The fusion rules for the spin field $\sigma$ which is part of the quasihole operator at level $k=3$ (see [6]), can be encoded in a Bratteli diagram with the same structure as the diagram 1 (note that the fields differ, of course). This is a consequence of the rank-level duality $\mathfrak{s u}(2)_{3} \leftrightarrow \mathfrak{s u}(3)_{2}$ (see $\S 16.6$ in [27). Thus the total intrinsic degeneracy for the $k=3$ Read-Rezayi state with $n$ quasiholes is given by $d_{n}=\mathcal{F}(n-2)$. Indeed, by summing the symbols $\left\{\begin{array}{c}n \\ F\end{array}\right\}_{3}$ over $F$, this result is reproduced.

The angular momentum multiplets can be found in the same way as described in section 7 . Let us note that for $k=1$ the only degeneracy factor remaining is $\left(\begin{array}{c}N+n \\ n\end{array}\right)$, which is precisely the orbital factor for the Laughlin states with quasiholes present. This was of course to be expected, as the $k=1$ Read-Rezayi states are in fact the Laughlin states.

To conclude the discussion on the counting for the Read-Rezayi states, we would like to mention that the numerical studies as presented for $k=3$ in [9] are in complete agreement with the counting formulas. At this point, no numerical results are available for $k \geq 4$. In the following section we will turn our attention to the counting of the NASS states for general level.

\section{Counting formulas for the NASS states}

In this section, we describe the counting for the NASS states at general level $k$. We will closely follow the procedure of the previous sections, that is, we start by writing down the chiral character corresponding to the $\mathfrak{s u}(3)_{k} / \mathfrak{u}(1)^{2}$ parafermions [18, 24]

$$
\operatorname{ch}\left(x_{1}, x_{2} ; q, k\right)=\sum_{a_{i}, b_{j}} \frac{q^{\frac{1}{2}\left(\mathbf{a} \cdot \mathbb{C}_{\mathbf{k}-\mathbf{1}} \cdot \mathbf{a}+\mathbf{b} \cdot \mathbb{C}_{\mathbf{k}-\mathbf{1}} \cdot \mathbf{b}-\mathbf{a} \cdot \mathbb{C}_{\mathbf{k}-\mathbf{1}} \cdot \mathbf{b}\right)}}{\prod_{i, j}(q)_{a_{i}}(q)_{b_{j}}} x_{1}^{i a_{i}} x_{2}^{j b_{j}},
$$


where we used the same notation as in eq. (37). This character is of the UCPF form with the $\mathrm{K}$-matrix equal to the K-matrix of the $\mathfrak{s u}(3)_{k} / \mathfrak{u}(1)^{2}$ parafermions: $\mathbb{K}=\left(\begin{array}{cc}2 & -1 \\ -1 & 2\end{array}\right) \otimes \mathbb{A}_{k-1}^{-1}$ [17]. The recursion relations corresponding to the basis of this theory can be written in the following way

$$
\begin{aligned}
Y_{(\mathbf{1}, \mathbf{m})}\left(x_{1}, x_{2} ; q, k\right) & =Y_{\left(\mathbf{1}-\mathbf{e}_{i}, \mathbf{m}\right)}+x_{1}^{i} q^{l_{i}-\frac{i(k-i)}{k}} Y_{\left(\mathbf{l}-\mathbb{C}_{k-1} \cdot \mathbf{e}_{i}, \mathbf{m}+\frac{1}{2} \mathbb{C}_{k-1} \cdot \mathbf{e}_{i}\right)} \\
Y_{(\mathbf{1}, \mathbf{m})}\left(x_{1}, x_{2} ; q, k\right) & =Y_{\left(\mathbf{1}, \mathbf{m}-\mathbf{e}_{j}\right)}+x_{2}^{j} q^{m_{j}-\frac{j(k-j)}{k}} Y_{\left(\mathbf{1}+\frac{1}{2} \mathbb{C}_{k-1} \cdot \mathbf{e}_{j}, \mathbf{m}-\mathbb{C}_{k-1} \cdot \mathbf{e}_{j}\right)} .
\end{aligned}
$$

Once again, we solve the recursion relations by matching these recursions to eq. (30). The truncated characters take the form

$$
\left.\begin{array}{rl}
Y_{(\mathbf{1}, \mathbf{m})}\left(x_{1}, x_{2} ; q, k\right)= & \sum_{a_{i}, b_{j}} q^{\frac{1}{2}\left(\mathbf{a} \cdot \mathbb{C}_{\mathbf{k}-\mathbf{1}} \cdot \mathbf{a}+\mathbf{b} \cdot \mathbb{C}_{\mathbf{k}-\mathbf{1}} \cdot \mathbf{b}-\mathbf{a} \cdot \mathbb{C}_{\mathbf{k}-\mathbf{1}} \cdot \mathbf{b}\right)} \times \\
& \prod_{i=1}^{k-1} x_{1}^{i a_{i}}\left[\left(\mathbf{l}+\left(\mathbb{I}_{k-1}-\mathbb{C}_{k-1}\right) \cdot \mathbf{a}+\frac{1}{2} \mathbb{C}_{k-1} \cdot \mathbf{b}\right)_{i}\right] \times \\
a_{i}
\end{array}\right] .
$$

We continue by specifying the parameters $l_{i}$ and $m_{j}$. We have to use the same construction as in the RR case, with the difference that we now need the number of spin-up and down particles (denoted by $n_{\uparrow}$ and $n_{\downarrow}$ ) created by the excess flux. Using $l_{i}=\frac{i n_{\uparrow}}{k}$ and $m_{j}=\frac{j n_{\downarrow}}{k}$ results in

$$
\begin{aligned}
& Y_{\left(n_{\uparrow}, n_{\downarrow}\right)}\left(x_{1}, x_{2} ; q, k\right)= \sum_{a_{i}, b_{j}} q^{\frac{1}{2}\left(\mathbf{a} \cdot \mathbb{C}_{\mathbf{k}-1} \cdot \mathbf{a}+\mathbf{b} \cdot \mathbb{C}_{\mathbf{k}-\mathbf{1}} \cdot \mathbf{b}-\mathbf{a} \cdot \mathbb{C}_{\mathbf{k}-\mathbf{1}} \cdot \mathbf{b}\right)} \times \\
& \prod_{i=1}^{k-1} x_{1}^{i a_{i}}\left[\frac{i n_{\uparrow}}{k}+\left(\left(\mathbb{I}_{k-1}-\mathbb{C}_{k-1}\right) \cdot \mathbf{a}+\frac{1}{2} \mathbb{C}_{k-1} \cdot \mathbf{b}\right)_{i}\right] \times \\
& a_{i} \\
& \prod_{j=1}^{k-1} x_{2}^{j b_{j}}\left[\frac{j n_{\downarrow}}{k}+\left(\left(\mathbb{I}_{k-1}-\mathbb{C}_{k-1}\right) \cdot \mathbf{b}+\frac{1}{2} \mathbb{C}_{k-1} \cdot \mathbf{a}\right)_{j}\right] . \\
& b_{j}
\end{aligned}
$$

From eq. (49) we obtain the symbols $\left\{\begin{array}{lll}n_{\uparrow} & n_{\downarrow} \\ F_{1} & F_{2}\end{array}\right\}_{k}$ by taking the limit $q \rightarrow 1$ of the coefficient of $x_{1}^{F_{1}} x_{2}^{F_{2}}$

$$
\begin{aligned}
& \left\{\begin{array}{ll}
n_{\uparrow} & n_{\downarrow} \\
F_{1} & F_{2}
\end{array}\right\}_{k}=\sum_{\substack{\sum i a_{i}=F_{1} \\
\sum j b_{j}=F_{2}}} \prod_{i=1}^{k-1}\left(\begin{array}{c}
\frac{i n_{\uparrow}}{k}+\left(\left(\mathbb{I}_{k-1}-\mathbb{C}_{k-1}\right) \cdot \mathbf{a}+\frac{1}{2} \mathbb{C}_{k-1} \cdot \mathbf{b}\right)_{i} \\
a_{i}
\end{array}\right) \times \\
& \prod_{j=1}^{k-1}\left(\begin{array}{c}
\frac{j n_{\uparrow}}{k}+\left(\left(\mathbb{I}_{k-1}-\mathbb{C}_{k-1}\right) \cdot \mathbf{b}+\frac{1}{2} \mathbb{C}_{k-1} \cdot \mathbf{a}\right)_{j} \\
b_{j}
\end{array}\right) .
\end{aligned}
$$

We know have specified all the ingredients of the counting formula for the NASS states

$$
\#_{\mathrm{NASS}}\left(N, \Delta N_{\phi}, k\right)=\sum_{N_{\uparrow, \downarrow}, n_{\uparrow, \downarrow}, F_{1,2}}^{\prime}\left\{\begin{array}{ll}
n_{\uparrow} & n_{\downarrow} \\
F_{1} & F_{2}
\end{array}\right\}_{k}\left(\begin{array}{c}
\frac{N_{\uparrow}-F_{1}}{k}+n_{\uparrow} \\
n_{\uparrow}
\end{array}\right)\left(\begin{array}{c}
\frac{N_{\downarrow}-F_{2}}{k}+n_{\downarrow} \\
n_{\downarrow}
\end{array}\right),
$$

where the prime on the sum indicates the constraints $N_{\uparrow}+N_{\downarrow}=N, n_{\uparrow}+n_{\downarrow}=2 k \Delta N_{\phi}$ and $N_{\uparrow}-N_{\downarrow}=n_{\downarrow}-n_{\uparrow}$. The last constraint is a necessary condition for the state to be a spin-singlet (for more information on the constraints, see 10]).

The case $k=2$ was explicitly discussed in section 7. For $k=1$ only the orbital degeneracy factors remain, and we obtain the counting formula for a particular class of Halperin states 28]. Indeed, for $k=1$, the NASS states reduce to the spin-singlet Halperin states. As already mentioned 
in section 7, the counting formula (51) with the symbols (50) exactly reproduces the results of the diagonalization studies for $k=2$ [10]. For $k \geq 3$, no numerical results are available at the moment.

\section{Discussion}

In this paper, we explained in which way parafermions can be used to define clustered quantum Hall states. The statistics of these parafermions is needed to understand the energy spectrum of the clustered states in the presence of quasiholes, as obtained from a numerical diagonalization study. We obtained explicit formulas for the symbols \{\}$_{k}$ for two classes of clustered states, needed in the counting formulas. From these formulas, also the spin and angular momentum multiplet structure of the quasihole degeneracies can be extracted.

One can say that the parafermion statistics is a crucial part in the understanding of the ground state properties of a system of interacting electrons (via an ultra local interaction) on the sphere in the presence of a magnetic field. In fact, we know of no other way in which this energy spectrum can be understood. The observation that ground state properties of a system of interacting electrons needs the knowledge of parafermion statistics is interesting by itself.

Recently, another class of paired (clustered) spin-singlet states was proposed [29]. An interesting property of these states is that the fundamental excitations over these states show a separation of the spin and charge degrees of freedom. We believe it should be possible to repeat the present analysis for these newly proposed states, though it will be more difficult, because the underlying parafermions are related to $\mathfrak{s o}(5)$, a non simply-laced Lie algebra. We leave this as a challenge for future work.

Acknowledgments. The author thanks P. Bouwknegt, R.A.J. van Elburg, N. Read, E. Rezayi and K. Schoutens for discussions. This research is supported in part by the foundation FOM of the Netherlands.

\section{REFERENCES}

[1] R.B. Laughlin, Anomalous quantum Hall effect: an incompressible quantum fluid with fractionally charged excitations, Phys. Rev. Lett. 50, 1395 (1983).

[2] G. Moore and N. Read, Nonabelions in the fractional quantum Hall effect, Nucl. Phys. B360, 362 (1991).

[3] R.L. Willett, J.P. Eisenstein, H.L. Störmer, D.C. Tsui, A.C. Gossard, and J.H. English, Observation of an even-denominator quantum number in the fractional quantum Hall effect, Phys. Rev. Lett. 59, 1776 (1987).

[4] W. Pan, J.-S. Xia, V. Shvarts, D.E. Adams, H.L. Störmer, D.C. Tsui, L.N. Pfeiffer, K.W. Baldwin, and K.W. West, Exact quantization of the even-denominator fractional quantum Hall state at $\nu=5 / 2$ Landau level filling factor, Phys. Rev. Lett. 83, 3530 (1999), cond-mat/9907356].

[5] N. Read. Paired fractional quantum Hall states and the $\nu=5 / 2$ puzzle, Physica B298, 121 (2001), [condmat/0011338.

[6] N. Read and E. Rezayi, Beyond paired quantum Hall states: parafermions and incompressible states in the first excited Landau level, Phys. Rev. B59, 8084 (1999), cond-mat/9809384.

[7] E. Ardonne and K. Schoutens, New class of non-abelian spin-singlet quantum Hall states, Phys. Rev. Lett. 82, 5096 (1999), cond-mat/9811352.

[8] N.R. Cooper, N.K. Wilkin, and J.M.F. Gunn. Quantum phases of vortices in rotating Bose-Einstein condensates, Phys. Rev. Lett. 87, 120405 (2001), cond-mat/0107005.

[9] V. Gurarie and E. Rezayi, Parafermion statistics and quasihole excitations for the generalizations of the paired quantum Hall states, Phys. Rev. B61, 5473 (2000), cond-mat/9812288.

[10] E. Ardonne, N. Read, E. Rezayi, and K. Schoutens, Non-abelian spin-sinqlet quantum Hall states: wave functions and quasihole state counting, Nucl. Phys. B607, 549 (2001), cond-mat/0104250.

[11] D. Gepner, New conformal field theories associated with Lie algebras and their partition functions, Nucl. Phys. B290, 10 (1987).

[12] N. Read and E. Rezayi, Quasiholes and fermionic zero modes of paired fractional quantum Hall states: the mechanism for nonabelian statistics, Phys. Rev. B54, 16864 (1996), cond-mat/9609079.

[13] A. Cappelli, L.S. Georgiev, and I.T. Todorov, Parafermion Hall states from coset projections of abelian conformal theories, Nucl. Phys. B599, 499 (2001), [hep-th/0009229]. 
[14] P. Bouwknegt and K. Schoutens, Exclusion statistics in conformal field theory-generalized fermions and spinons for level-1 WZW theories, Nucl. Phys. B547, 501 (1999), hep-th/9810113.

[15] K. Schoutens, Exclusion statistics in conformal field theory spectra, Phys. Rev. Lett. 79, 2608 (1997), [condmat/9706166.

[16] A. Berkovich and B. McCoy, The universal chiral partition function for exclusion statistics, in Statistical Physics on the Eve of the 21st Century, Series on Adv. in Stat. Mech., Vol. 14, M.T. Batchelor and L.T. Wille, eds. (World Scientific, Singapore, 1999), pp. 240-256, hep-th/9808013].

[17] E. Ardonne, P. Bouwknegt, and P. Dawson, in preparation.

[18] A. Kuniba, T. Nakanishi, and J. Suzuki, Characters in conformal field theories from thermodynamic Bethe Ansatz, Mod. Phys. Lett. A8, 1649 (1993), hep-th/9301018.

[19] E. Ardonne, P. Bouwknegt, and K. Schoutens, Non-abelian quantum Hall states - Exclusion statistics $K$ matrices and duality, J. Stat. Phys. 102, 421 (2001), cond-mat/0004084.

[20] P. Bouwknegt, Multipartitions, generalized Durfee squares and affine Lie algebra characters, J. Aust. Math. Soc. A, to appear, math.CO/0002223].

[21] G.E. Andrews, R.J. Baxter, and P.J. Forrester, Eight-vertex SOS model and generalized Rogers-Ramanujantype identities, J. Stat. Phys. 35, 193 (1984).

[22] A.B. Zamolodchikov and V.A. Fateev, Nonlocal (parafermion) currents in two-dimensional conformal quantum field theory and self-dual critical points in $Z_{N}$-symmetric statistical systems, Sov. Phys. JETP 62, 215 (1985).

[23] J. Lepowski and M. Primc, Structure for standard modules of the affine Lie algebra $A_{1}^{(1)}$, Contemporary Mathematics, vol. 46, AMS, Providence, 1985.

[24] G. Georgiev. Combinatorial constructions of modules for infinite-dimensional Lie algebras, II. Parafermionic space, q-alg/9504024.

[25] S. Guruswamy and K. Schoutens, Non-abelian exclusion statistics, Nucl. Phys. B556, 530 (1999), condmat/9903045.

[26] E. Ardonne, P. Bouwknegt, S. Guruswamy, and K. Schoutens, K-matrices for non-abelian quantum Hall states, Phys. Rev. B61, 10298 (2000), cond-mat/9908285.

[27] P. Di Francesco, P. Mathieu, and D. Sénéchal, Conformal field theory, Springer, New York, 1997.

[28] B. Halperin, Theory of the quantized Hall conductance, Helv. Phys. Acta 56, 75 (1983).

[29] E. Ardonne, F.J.M. van Lankvelt, A.W.W. Ludwig, and K. Schoutens, Separation of spin and charge in paired spin-singlet quantum Hall states, cond-mat/0102072.

Institute for Theoretical Physics, University of Amsterdam, Valckenierstraat 65, 1018 Xe AmsterDAM, THE NETHERLANDS

E-mail address: ardonne@science.uva.nl 